\section{Review}

Correspondence

Taghrid Istivan

taghrid.istivan@rmit.edu.au

\title{
Phospholipase A in Gram-negative bacteria and its role in pathogenesis
}

\author{
Taghrid S. Istivan and Peter J. Coloe \\ Biotechnology and Environmental Biology, School of Applied Sciences, RMIT University, \\ Bundoora West Campus, PO Box 71, Bundoora, Melbourne, VIC 3083, Australia
}

\begin{abstract}
Phospholipase A (PLA) is one of the few enzymes present in the outer membrane of Gram-negative bacteria, and is likely to be involved in the membrane disruption processes that occur during host cell invasion. Both secreted and membrane-bound phospholipase $A_{2}$ activities have been described in bacteria, fungi and protozoa. Recently there have been increasing reports on the involvement of PLA in bacterial invasion and pathogenesis. This review highlights the latest findings on PLA as a virulence factor in Gram-negative bacteria.
\end{abstract}

\section{Introduction}

Phospholipases constitute a diverse subgroup of lipolytic enzymes that share the ability to hydrolyse one or more ester linkages in phospholipids, with phosphodiesterase as well as acyl hydrolase activity. The amphipathic nature of phospholipids creates obstacles for the enzymes, as the substrates are assembled into bilayers or micelles and are not present in significant amounts as a single soluble substrate. Although all phospholipases target phospholipids as substrates, they vary in the site of action on the phospholipid molecule, their function and mode of action, and their regulation (Waite, 1996). Phospholipases function in various roles, ranging from the digestion of nutrients to the formation of bioactive molecules. This diversity of function suggests that phospholipases are critical to life; the continual remodelling of cell membranes requires the action of one or more phospholipases. The most common phospholipids in mammalian cells are phosphatidylcholine (PC), phosphatidylserine (PS), phosphatidylinositol (PI) and phosphatidylethanolamine (PE). The plasma membrane of most eukaryotic cells contains predominantly PC and sphingomyelin in the outer leaflet, and PI, PE and PS in the inner leaflet. Therefore, an extracellular phospholipase would not be expected to lyse a target cell unless the outer-leaflet phospholipids were efficiently hydrolysed substrates of the enzyme (Schmiel \& Miller, 1999). In contrast to mammalian cells, Escherichia coli membranes are composed of PE with some phosphatidylglycerol (PG), cardiolipin and trace amounts of PS.

Invasion of host cells by most pathogens requires penetration and damage of the cell membrane, which is mediated by either physical or enzymic means, or a combination of the two. Phospholipids and proteins represent the major chemical constituents of the host cell envelope. Therefore phospholipases are likely to be involved in the membrane disruption processes that often occur during host cell invasion (Waite, 1996), such as the $\mathrm{pH}$-activated phospholipase $\mathrm{A}_{2}$ of Helicobacter pylori (Berstad et al., 2002).

\section{Classification of phospholipases}

The classification of phospholipases is based on their site of attack on the phospholipid. Qualifying letters, such as A, B, $\mathrm{C}$ and $\mathrm{D}$, are used to differentiate the phospholipases and to indicate the specific bond targeted in the phospholipid molecule. Phospholipases A are acyl hydrolases classified according to their site of action upon the hydrolysis of the acyl ester bond. Phospholipase $\mathrm{A}_{1}\left(\mathrm{PLA}_{1}\right)$ hydrolyses the fatty acyl ester bond at the $s n-1$ position of the glycerol moiety, while phospholipase $\mathrm{A}_{2}\left(\mathrm{PLA}_{2}\right)$ removes the fatty acid at the $s n-2$ position of this molecule (Fig. 1a). The action of $\mathrm{PLA}_{1}$ and $\mathrm{PLA}_{2}$ results in the accumulation of free fatty acids and 2-acyl lysophospholipid or 1-acyl lysophospholipid, respectively. The fatty acid, which is still linked to the lysophospholipid, is, in turn, cleaved by other enzymes termed lysophospholipases (Fig. 1b). Some phospholipases hydrolyse both acyl groups and are termed phospholipases $\mathrm{B}$; they also have high lysophospholipase activity.

\section{Bacterial phospholipase A}

Phospholipase $A_{1}\left(P L A_{1}\right)$. The phospholipases $A_{1}$ in general constitute a large group of 1-acyl hydrolases, some of which also degrade neutral lipids (lipases). PLA ${ }_{1}$ has been reported to play an important role as a virulence factor in some bacteria and fungi. Schmiel et al. (1998) reported on the yplA gene, which encodes secreted PLA activity, linked to lecithin-dependent haemolysis, in the human pathogen Yersinia enterocolitica. This yplA gene product has $74 \%$ identity and $85 \%$ similarity to the PLA found in Serratia liquefaciens (Givskov et al., 1988). It was also reported that this PLA $_{1}$ activity plays a role in the pathogenesis of $Y$. enterocolitica and in the following inflammatory process observed in humans and mice, by promoting colonization and increasing the pathological changes resulting from the host inflammatory response (Schmiel et al., 1998). 
(a)

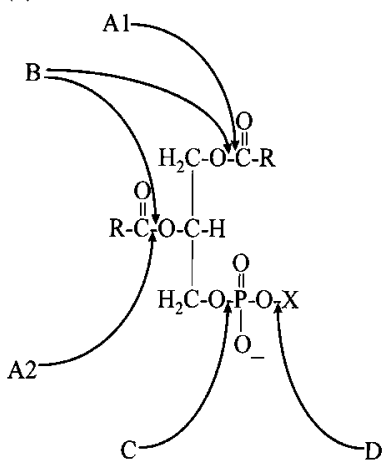

(b)

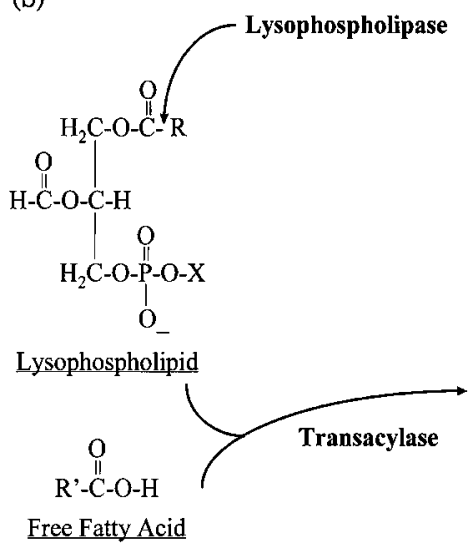

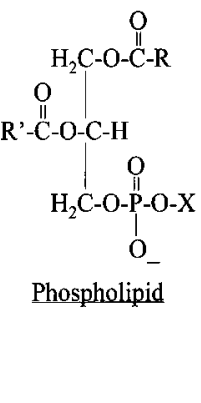

Fig. 1. Sites of action of various phospholipases. (a) $\mathrm{A} 1$ and $\mathrm{A} 2, \mathrm{PLA}_{1}$ and $\mathrm{PLA}_{2}$, respectively; B, PLB; C, PLC; D, PLD. (b) Lyso-PL and Lyso-PL transacylase (Ghannoum, 2000; reproduced with permission from the publisher: http://www.asm.org/).
In addition to its physiological roles, $\mathrm{PLA}_{1}$ is of particular interest for industrial applications as it yields 2-acyl lysophospholipids, which are excellent emulsifiers suitable for use in the food technology, cosmetics and pharmaceutical industries (Song \& Rhee, 2000). Secreted (extracellular) $\mathrm{PLA}_{1}$ activity from Serratia sp. strain MK1 isolated from Korean soil was purified and characterized by Kim \& Rhee (1996). The cloning and characterization of the $p l d A_{1}$ gene, encoding a phospholipase with a predicted molecular mass of $33.4 \mathrm{kDa}$, revealed a significant similarity (around $70 \%$ ) to $\mathrm{PLA}_{1}$ from S. liquefaciens and Y. enterocolitica (Givskov et al., 1988; Schmiel et al., 1998). Furthermore, improving the conditions for a large-scale production of a soluble and active enzyme from Serratia sp. MK1 in E. coli, which was further improved by evolutionary molecular engineering to increase the thermostability of the produced enzyme, has broadened the possibility of the application of this $\mathrm{PLA}_{1}$ in various phospholipid-related industries (Song et al., 1999; Song \& Rhee, 2000).

Two phospholipases with $\mathrm{PLA}_{1}$ activity have been purified from $E$. coli based on their differential sensitivity to treatment with detergents. The first is a detergent-resistant enzyme, of the outer membrane (OMPLA), with broad substrate specificity, hydrolysing all phospholipids and many neutral glycerides (Rock et al., 1996). This enzyme is unusually resistant to inactivation by heat and ionic detergents and requires calcium for maximal activity. The second enzyme is a detergent-sensitive $\mathrm{PLA}_{1}$ found as a soluble fraction on the cytoplasmic membrane. This cytoplasmic $\mathrm{PLA}_{1}$, with a high degree of specificity for phosphatidylglycerol, can be inactivated by heat and by ionic detergents. This enzyme also acts as a transacylase and requires calcium for activity (Rock et al., 1996).

Phospholipase $\mathbf{A}_{2}$. The eukaryotic phospholipases $A_{2}$ $\left(\mathrm{PLA}_{2}\right)$ were the first of the phospholipases to be recognized. The pancreatic phospholipase has been known to degrade phosphatidylcholine since 1878, and at the turn of the century, cobra venom was shown by Keyes in 1902 to have haemolytic activity directed toward the membranes of erythrocytes (Waite, 1996). The family of eukaryotic
$\mathrm{PLA}_{2}$ enzymes is diverse and has been shown to function in digestion of lipids, microbial degradation, inflammation, cell signalling and membrane remodelling. PLA proteins are of great interest to the pharmaceutical industry since they are responsible for the release of arachidonic acid from membranes, and the subsequent conversion of this fatty acid to leukotrienes and prostaglandins is part of the inflammatory response (Farber et al., 1999).

Secreted and membrane-bound $\mathrm{PLA}_{2}$ activity has been described in bacteria, fungi and protozoa (Waite, 1996; Ghannoum, 2000; Matoba et al., 2002). This activity is reported to be calcium-dependent and related to pathogenesis in most micro-organisms. The crystal structure and the tertiary structure of the secreted $\mathrm{PLA}_{2}$ (EC 3.1.1.4) from Streptomyces violaceoruber A-2688 have been determined by NMR and X-ray analyses. Interestingly, the X-ray diffraction data of this $\mathrm{PLA}_{2}$ indicated that although the tertiary structure of the microbial $\mathrm{PLA}_{2}$ is strikingly different from that of the bovine pancreatic phospholipase, the internal motion, which is associated with the calcium binding, phospholipid binding and allosteric interfacial activation, is common to both (Matoba et al., 2002; Matoba \& Sugiyama, 2003).

\section{Outer-membrane PLA in Gram-negative bacteria}

Molecular and biochemical characteristics of the enzyme. Outer-membrane PLA (OMPLA; EC 3.1.1.32) is one of the few enzymes present in the outer membrane of Gram-negative bacteria. PLA and lysophospholipase activities were first reported in E. coli in 1971 and the membrane-bound PLA activity was isolated and purified later in the same year (Scandella \& Kornberg, 1971; Doi et al., 1972). OMPLA of E. coli, the most-studied protein among this group of phospholipases, is a $31 \mathrm{kDa}$ protein encoded by the structural gene pldA; the amino acid sequence is composed of 269 amino acids residues preceded by a 20 amino acid signal sequence which targets the protein across the inner membrane. The gene is under the control of a constitutive promoter, resulting in the presence of 500 copies of the enzyme in the outer membrane of 
normally growing cells (de Geus et al., 1983; Homma et al., 1984). This enzyme is strictly calcium dependent and displays broad substrate specificity. It hydrolyses the acyl ester bonds in phospholipids with PLA $\mathrm{A}_{1}$ and $\mathrm{A}_{2}$ activities, and also has 1-acyl and 2-acyl lysophospholipase activity, as well as mono- and diacylglyceride lipase activities (Horrevoets et al., 1989).

Brok et al. (1994) suggested that OMPLA is not required for the bacterial cell under laboratory conditions but it might be essential for growth in the natural environment. Kingma \& Egmond (2002a) suggested a function for OMPLA as a housekeeping enzyme, indicating that the inhibition of OMPLA by its substrate provides the means to prevent this enzymic activity from being lethal to the cell once activated. Another speculative function of OMPLA is related to organic solvent tolerance in the bacterium; however, the role of OMPLA in this process needs to be clarified (Snijder \& Dijkstra, 2000).

Merck et al. (1997) used genetic approaches to propose a model for the topology of OMPLA in Salmonella typhimurium based on the prediction of turns and loops and calculations of hydrophobicity and hydrophobic moment. In this model, OMPLA traverses the outer membrane 14 times as antiparallel amphipathic $\beta$-strands, thereby exposing hydrophilic loops to the cell surface, while the $\beta$-strands are connected by short turns on the periplasmic side. A similar $\beta$-barrel structure, but only involving 12 strands, was reported for OMPLA in E. coli by Brok et al. (1994).

Activation and functions of OMPLA. Although OMPLA of Gram-negative bacteria is an integral membrane protein that is embedded in its own substrate in the cell envelope, no enzymic activity can be detected in normally growing cells. Genetic regulation cannot explain the absence of enzymic activity because OMPLA is constitutively expressed, and the expressed protein is correctly transported to and inserted into the outer membrane, which is composed of phospholipids in the inner leaflet and of lipopolysaccharide (LPS) in the outer leaflet. As uncontrolled breakdown of the outer membrane would have lethal consequences for the cell, in vivo, OMPLA is present in the outer membrane as a monomeric protein in a dormant state. Hence, enzymic activity can only be induced after severe perturbation of the cell envelope integrity, which occurs during various processes such as phage-induced lysis, temperature shock and colicin secretion. Membrane-perturbing peptides, such as polymyxin $\mathrm{B}$, melittin or cardiotoxin, can also activate the enzyme (Dekker, 2000; Snijder \& Dijkstra, 2000).

Structural analysis of the OMPLA molecule. The $12-\beta$ stranded architecture of E. coli OMPLA resembles the folds of other $\beta$-barrel outer-membrane proteins (OMPs) that have 8-22 strands (Brok et al., 1994). The crystal structures of the monomeric and the inhibited dimeric enzymes were determined by Snijder et al. (1999); this revealed that OMPLA monomers are folded into a 12stranded antiparallel $\beta$-barrel with a convex side and a flat side with approximate dimensions of $20 \times 30 \times 45 \AA$ (Fig. 2). As reviewed by Dekker (2000), the $\beta$-strands are connected by long extracellular loops and short periplasmic turns. The interior of the $\beta$-barrel is hydrophilic, with extensive hydrogen-bonding networks and electrostatic interactions, resulting in a very stable structure. The $\beta$-barrel has a hydrophobic outer surface with which the protein is embedded in the outer membrane. The active site of the enzyme is located at the hydrophilic-hydrophobic boundary (apolar-polar boundary) of the outer leaflet of the outer membrane (Dekker, 2000).

Ser-144 and His-142 have been identified by chemical modification and by site-directed mutagenesis as essential residues in the active site, in an arrangement resembling the corresponding residues of a serine hydrolase catalytic triad. However, instead of the Asp or Glu that is normally present in the triad of serine hydrolases, a neutral asparagine (Asn156) was found in OMPLA (Kingma et al., 2000).

As reviewed by Snijder \& Dijkstra (2000), dimeric and monomeric OMPLA hardly exhibit structural differences, as OMPLA dimers are formed by the association of two enzyme monomers interacting via the flat barrel side (Fig. 2). Dimerization creates two extended clefts along the subunit interface, which run down from the two active sites. Thus, although the monomer contains a catalytic triad, the monomeric enzyme form lacks catalytic activity due to

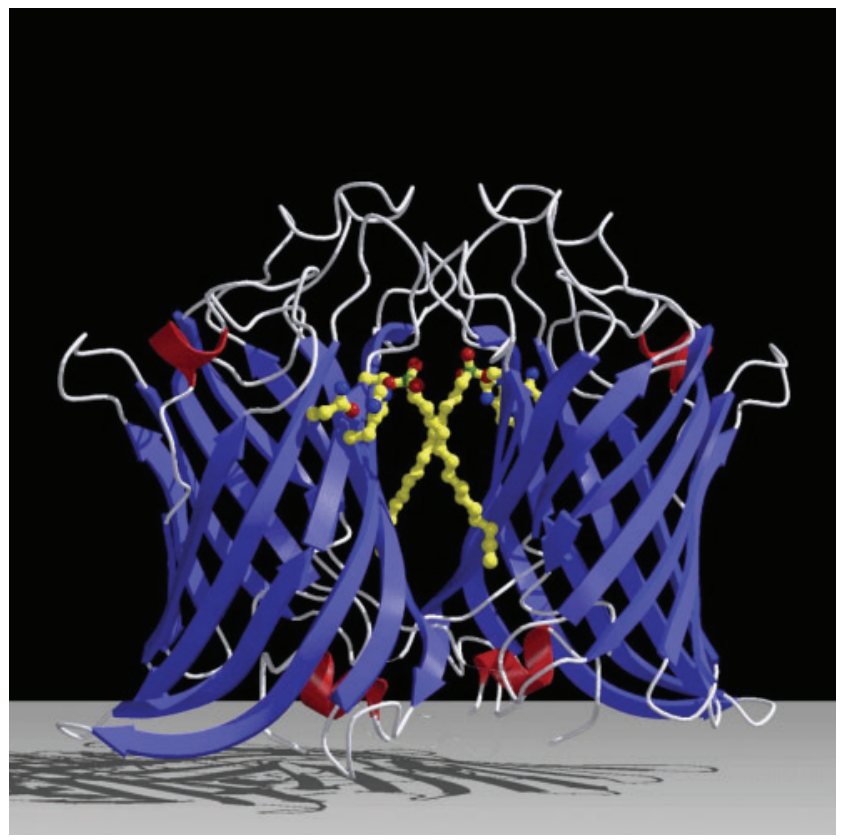

Fig. 2. 3D structure for the OMPLA molecule of $E$. coli in the active dimeric state (Snijder et al., 1999; reproduced with permission from the publisher: http://www.nature.com/). 
absence of substrate-binding pockets, which are only formed in the dimeric complex. The architecture of the catalytic site and substrate-binding clefts allows a broad range of substrates to bind; therefore, substrates with either one or two acyl chains of various lengths can be productively bound (Snijder \& Dijkstra, 2000).

Dimerization as an essential step for OMPLA activity. Snijder et al. (1999) proposed that bacterial phospholipids are only found in the inner leaflet of the outer membrane, where OMPLA is present in the inactive monomeric form, but that the enzyme will be activated when the bacterial membrane structure is disturbed. They suggested that the relief of lipid asymmetry and the presentation of substrate to the outer leaflet induces activation by promoting dimerization and calcium binding. Kingma \& Egmond (2002b) constructed a well-defined covalent OMPLA dimer to study the importance of dimerization for activity regulation, both in a detergent system and after reconstitution in a phospholipid bilayer. This study revealed that a proper OMPLA dimer displays high affinity for calcium as OMPLA variants with an impaired dimeric interface also lacked high-affinity calcium binding. The structure of the detergent in crystals of OMPLA was determined by Matoba \& Sugiyama (2003). Further research indicated that the enzyme's active site is positioned just outside the hydrophobic detergent zone; therefore, it is in a proper location to catalyse the hydrolysis of phospholipids in a natural membrane (Snijder et al., 2003). Baaden et al. (2003) studied the possible differences in conformational dynamics that may be related to enzyme activation. They indicated that dimeric OMPLA is less flexible than monomeric OMPLA, especially around the active site, and also proposed that the increased stability of the active site in dimeric OMPLA is a consequence of the local ordering of water around the nearby calcium ion.

Role of calcium in activation of OMPLA. OMPLA has two calcium-binding sites per monomer: one high-affinity site $\left(K_{\mathrm{d}} 36 \mu \mathrm{M}\right)$ and a binding site with a 10 -fold lower affinity $\left(K_{\mathrm{d}} 358 \mu \mathrm{M}\right)$ (Ubarretxena-Belandia et al., 1998). As dimerization occurs at low calcium concentration, Snijder \& Dijkstra (2000) suggested that the first calcium ion plays a role in dimer stabilization. However, in the colon, the normal habitat of E. coli, the calcium level exceeds the determined binding affinities for calcium to OMPLA. Therefore, it is unlikely that the requirement for the cofactor calcium is involved in the regulation of OMPLA activity in vivo (Dekker, 2000). One calciumbinding site of the OMPLA monomer is located approximately $10 \AA$ away from the active site and is bound between loops L3 and L4 (L3L4 site). After dimerization a second calcium-binding site (catalytic site or interfacial site) at the dimer interface is present (Fig. 3). The calcium ion is ligated by the side chain of Ser-152 and by one main-chain carbonyl oxygen atom from each monomer (Snijder \& Dijkstra, 2000; Dekker, 2000).
Crystallographic studies by Snijder et al. (2001) revealed that the L3L4 site was found only in the structure of monomeric OMPLA, whereas the interfacial site (catalytic site) was only found in the dimeric enzyme, with no calcium binding observed at the L3L4 site. This indicates that calcium binding occurs in the L3L4 site of OMPLA monomers. However, the calcium ion is displaced from this site when the dimers are formed, which indicates that calcium plays an important role in the formation of the active dimer from two inactive monomers. Furthermore, Snijder et al. (2001) suggested that the L3L4 site could serve as a sink for calcium ions, and that it might complex with the LPS matrix, thus maintaining the barrier function of the outer membrane. They also indicated that the key elements of this model (calcium binding and activation) are conserved in Gramnegative bacteria, suggesting that OMPLA architecture in this group is most likely the same, and that its activity is calcium- and dimerization-dependent. Several of the conserved residues are close in sequence, and form a complete and highly specific consensus sequence motif (YTQ- $\mathrm{X}_{\mathrm{n}}-\mathrm{G}-$ $\mathrm{X}_{2}-\mathrm{H}-\mathrm{X}-\mathrm{SNG}$ ), which proves to be much more specific than a motif based on the active-site residues alone as indicated in Fig. 4 (Snijder et al., 2001).

\section{Molecular analysis of OMPLA sequences encoded by the pldA gene in Gram-negative bacteria}

Brok et al. (1994) compared the predicted primary structures of the pldA gene in S. typhimurium, Klebsiella pneumoniae and Proteus vulgaris with that of the E. coli pldA gene. This sequence analysis revealed a high degree of homology, with $79 \%$ of the amino acid residues being identical in all four proteins. Furthermore, enzymically active gene products were expressed when these pldA genes were cloned in E. coli. In a subsequent publication Brok et al. (1998) screened databases of whole-genome sequencing projects, which revealed the presence of proteins with homology to OMPLA in H. pylori, Campylobacter jejuni, Yersinia pestis, Neisseria meningitidis and Neisseria gonorrhoeae. Comparison of OMPLA amino acid sequences in these bacteria also revealed that 30 amino acid residues are completely conserved, indicating an important physiological role of this enzyme in Gram-negative bacteria (Brok et al., 1998). However, in a following study which involved sequence alignment of complete OMPLA sequences in 16 different species of Gram-negative bacteria, Snijder et al. (2001) indicated that only 20 amino acid residues are absolutely conserved and another 17 are similar in the sequences of E. coli, Bordetella pertussis, Campylobacter coli, C. jejuni, Enterobacter agglomerans, H. pylori 2669551, H. pylori J99, Salmonella typhi, S. typhimurium, K. pneumoniae, N. gonorrhoeae, N. meningitidis, Pasteurella multocida, Proteus vulgaris, $Y$. pestis and $Y$. pseudotuberculosis, therefore yielding a homology of only $13.5 \%$ (Fig. 4 ). The study indicated that the best-conserved residues are the active-site residues His-142 and Ser-144 and also the residues Asn-145 and Gly-146 which participate in the oxyanion-hole 


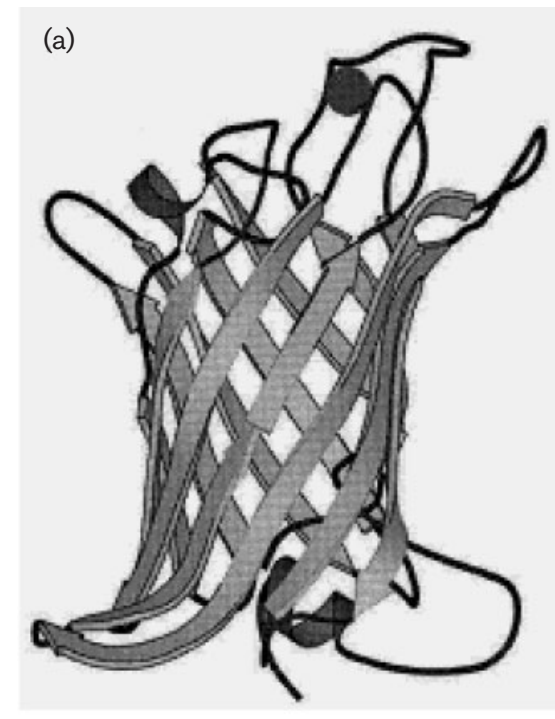

(I)

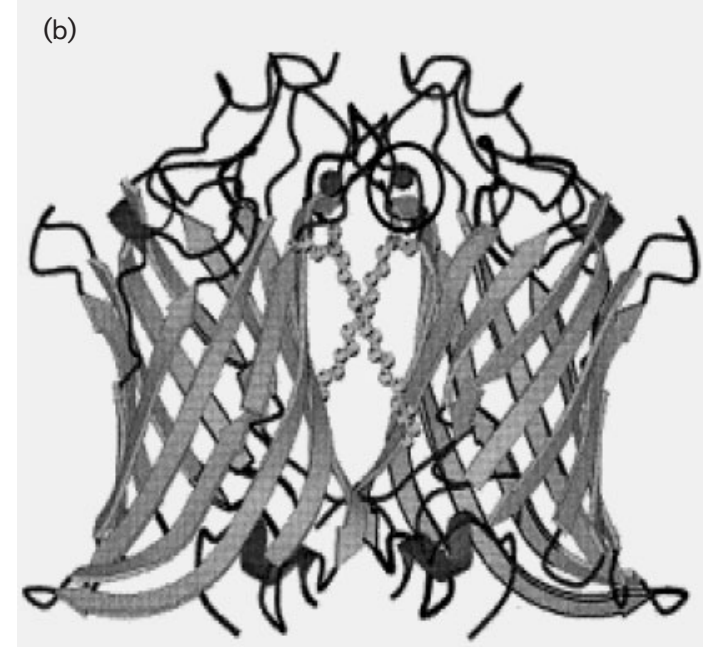

(I)

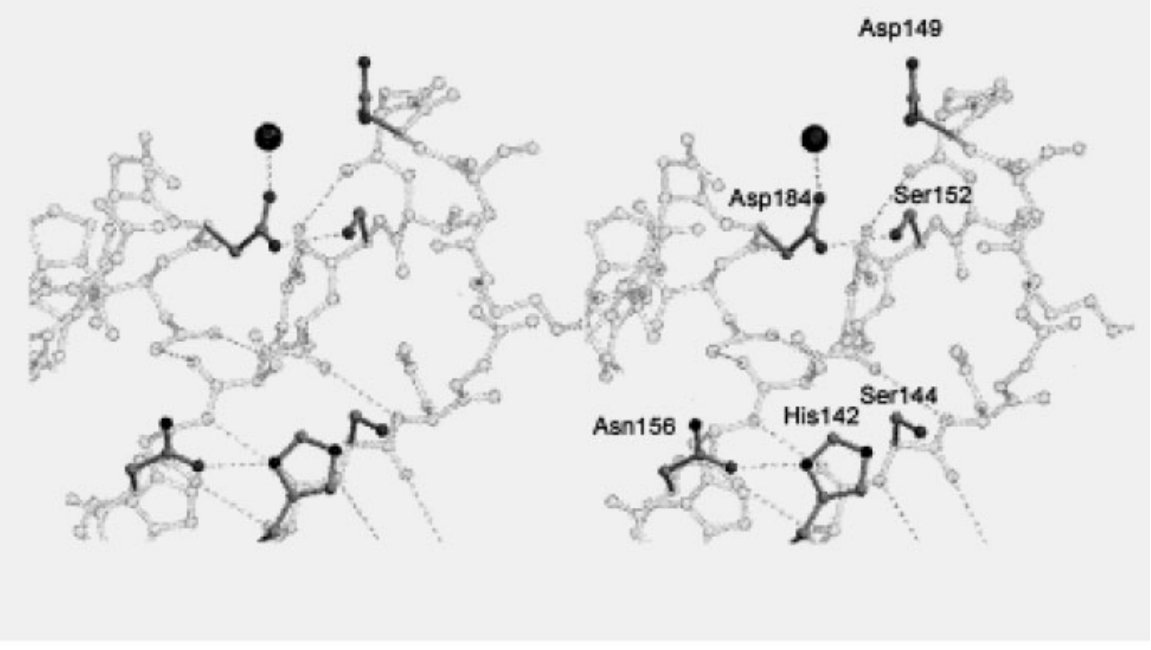

(II)
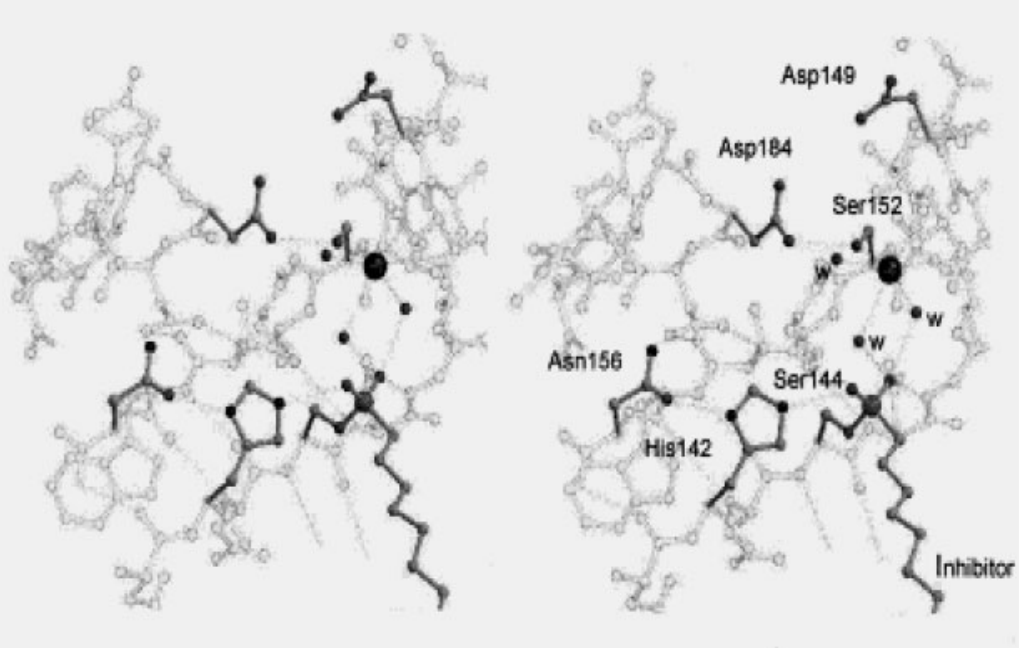

(II)

Fig. 3. Diagrams for calcium-binding sites in monomeric and dimeric molecules of E. coli OMPLA by Kingma et al. (2002) (reproduced with permission from the publisher: http://www.elsevier.com/). (a) The structure of monomeric OMPLA (I), with a calcium ion bound between a carboxylate oxygen atom of Asp-149 in loop 3 and a carboxylate oxygen atom of Asp-184 in loop 4 (II). (b) Crystal structure of inhibited, dimeric OMPLA with one calcium ion per monomer bound at the dimer interface (I). The calcium ion is coordinated by the hydroxyl group of Ser-152 and the Ser-106 carbonyl oxygen atom of one monomer, the Arg-147 carbonyl oxygen atom of the other monomer, and three water molecules (II).

formation. Furthermore, the catalytic calcium site is conserved among all OMPLA sequences, as Ser-152, which ligates the calcium ion with its hydroxyl group, is absolutely conserved in all Gram-negative bacteria investigated. In contrast, the L3L4 calcium site is not absolutely conserved, as Asp-149 is replaced by an arginine residue in Neisseria species, and by a glycine residue in the Helicobacter strains. The second calcium ligand, Asp-184, is conserved in all except in Helicobacter strains, where it is replaced by a serine residue (Snijder et al., 2001).
The biochemical relevance of OMPLA's calcium-binding sites was investigated in E. coli using site-directed mutagenesis (Kingma et al., 2002). The study concluded that the interfacial calcium site is essential for both catalysis and calcium-dependent dimerization, whereas the L3L4 site is redundant in vitro. These results are in accordance with sequence homology noted for several OMPLAs by Snijder et al. (2001), which revealed absolute conservation of Ser152, whereas Asp-149 is not conserved in Neisseria species, and neither Asp-149 nor Asp-184 is conserved in H. pylori 


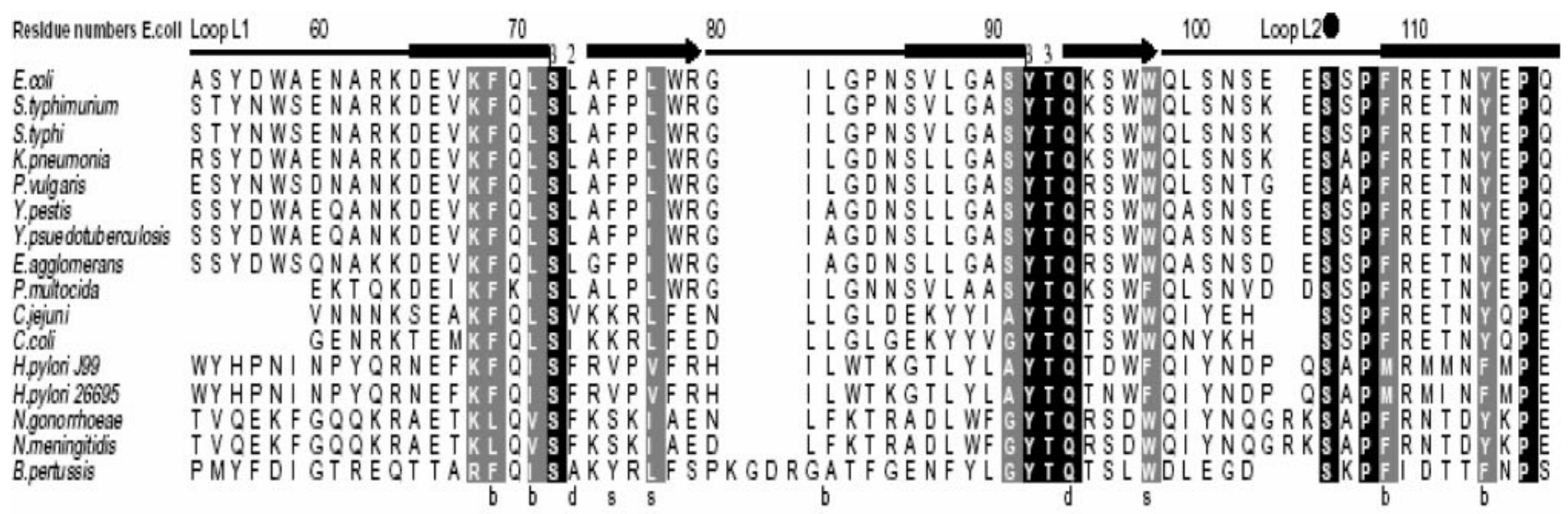

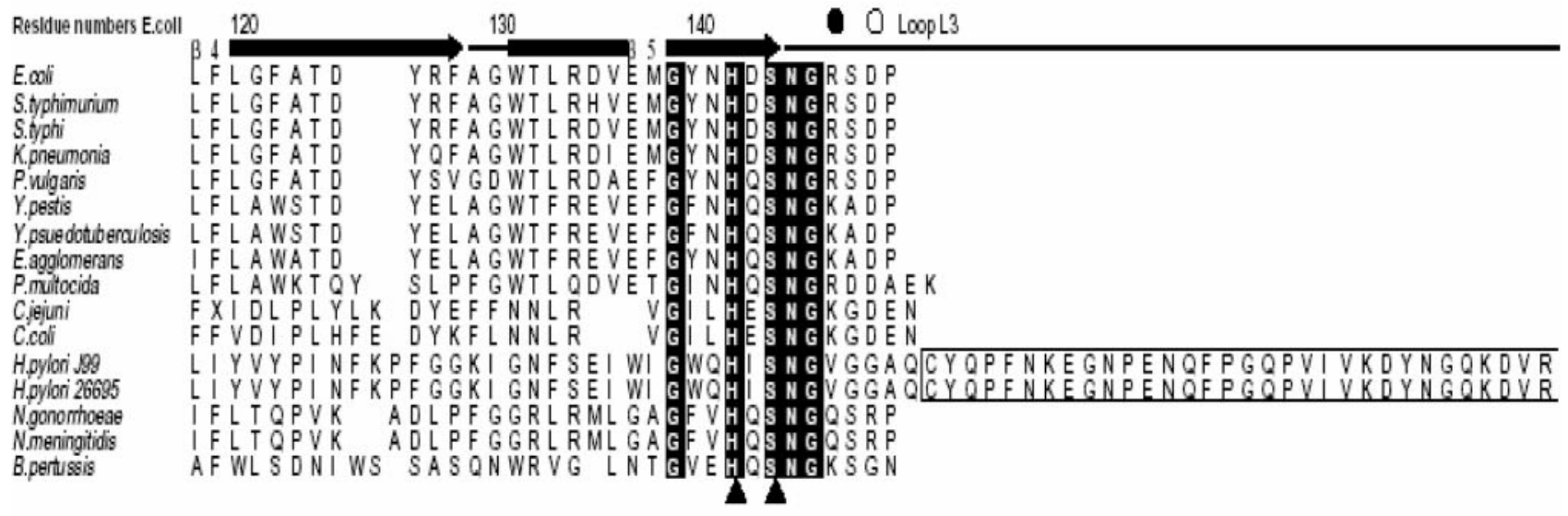

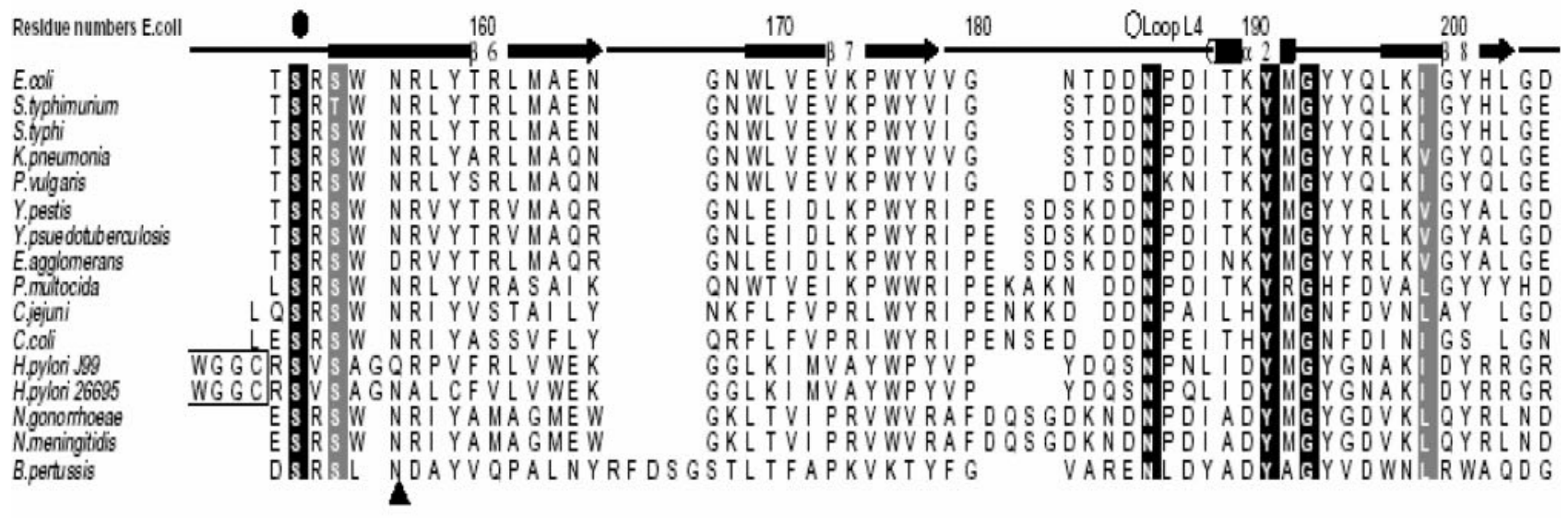

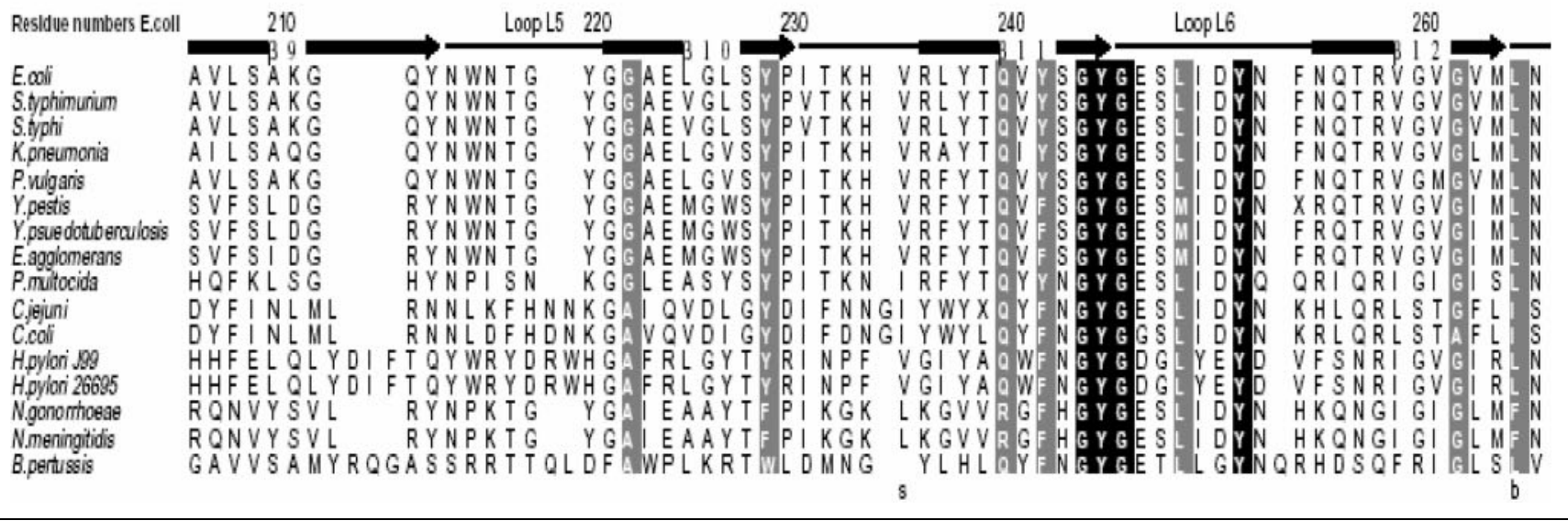


and B. pertussis. However, although OMPLA in H. pylori lacks the L3L4 calcium site, its activity still depends on the cofactor calcium (Kingma et al., 2002). The lack of the L3L4 calcium site may be related to differences in outermembrane composition compared to that of E. coli. In Neisseria spp., phospholipid molecules are present in the outer leaflet of the membrane, while the outer membrane of $H$. pylori may contain substantial amounts of lysophospholipids. Alternatively, those OMPLAs, which are least similar to the E. coli OMPLA, may still have an L3L4 site that is obscured by sequence differences and insertions (Snijder et al., 2001).

\section{Relationship between bacterial phospholipases and pathogenesis}

Phospholipase activity has been linked to pathogenesis in bacterial species which cause different disease syndromes such as the massive tissue destruction related to gas gangrene, skin and lung infections caused by Pseudomonas aeruginosa, and food-borne listeriosis (Schmiel \& Miller, 1999). However, although the importance of some bacterial phospholipases in pathogenesis is certain, the exact mechanism of the phospholipase action in vivo has not been definitively determined. Phospholipase toxicity has been linked to cytolytic activity resulting from the accumulation of membrane-destabilizing products or by the extensive destruction of membrane phospholipids. Cytolysis can be caused directly by a very active bacterial phospholipase with broad specificity or in concert with host degradative enzymes induced by the bacterial phospholipase. The cytolytic properties of the phospholipase are dependent on its ability to interact directly with and hydrolyse phospholipids in the membrane. Frequently, for convenience the lytic activity is measured as haemolysis, regardless of the likelihood of a particular pathogen causing systemic infection or a bacterial phospholipase entering the bloodstream (Schmiel \& Miller, 1999). Although phospholipases C (PLCs) are the best-studied bacterial phospholipases as virulence factors, various studies have indicated that many bacterial outermembrane or secreted phospholipases are cytolytic and therefore responsible for tissue damage during infection. Examples include $\mathrm{PLA}_{1}, \mathrm{~A}_{2}$ and $\mathrm{C}$ activities in Helicobacter pylori, which were linked to the degradation of the phospholipid components of the mucosal barrier, and the secreted PLA 1 activity of the human pathogen Y. enterocolitica (Nilius \& Malfertheiner, 1996; Songer, 1997; Schmiel et al., 1998).

\section{Phospholipase A as a virulence determinant in Gram-negative bacteria}

Helicobacter pylori. H. pylori possesses several different phospholipase activities, such as $\mathrm{PLA}_{1}, \mathrm{PLA}_{2}$ and PLC, which are linked to the degradation of the phospholipid components of the mucosal barrier. In addition to direct disruption of the mucosal barrier, phospholipases are also able to initiate production of leukotrienes and other eicosanoids, converted from arachidonic acid, which could also affect membrane permeability and mucous discharge (Nilius \& Malfertheiner, 1996). The H. pylori 26695 and J99 genome sequences revealed the presence of an open reading frame (HP0499) encoding a putative protein of around $42 \cdot 5 \mathrm{kDa}$ with homology $(54 \cdot 1 \%$ similarity) to $E$. coli OMPLA; the gene was confirmed to encode an active phospholipase with lecithinase, $\mathrm{PLA}_{2}$, and haemolytic activities (Dorrell et al., 1999). The researchers constructed an isogenic pldA mutant in $H$. pylori, which showed a marked reduction in $\mathrm{PLA}_{2}$ and haemolytic activities and, unlike the wild-type, was unable to colonize mice at 2 and 8 weeks. Although the ability of the mutant to adhere to human gastric adenocarcinoma cells was unaffected, it did induce a significant immune response, indicating a role for PLA in colonization of the gastric mucosa and possible tissue damage after colonization (Dorrell et al., 1999). These findings were confirmed by Zhang et al. (2002); they indicated that mutations in genes encoding the urease (ureB) and phospholipase (pldA) enzymes did not affect adherence of the bacteria to gastric epithelial cell lines. However, adherence to gastric epithelial cells in vitro does not imply that the mutant can successfully colonize in vivo.

Xerry \& Owen (2001), in a study to determine the degree of variation in the PLA gene in H. pylori isolates, found that the pldA gene is a highly conserved feature of the $H$. pylori genome irrespective of the geographical origin of the isolate. The study also investigated links between pldA genotype and clinical disease severity, as well as with variation in cagA status and vacA genotypes. It was concluded that pldA is a conserved feature of the $H$. pylori genome, with isolates of the same PCR-RFLP genotype having a widespread geographical distribution. The study also supported the theory that the role for PLA activity in $H$. pylori is in colonization and persistence rather than in chronicity of infection (Xerry \& Owen, 2001).

H. pylori can spontaneously and reversibly change its membrane lipid composition, under slightly acidic conditions,

Fig. 4. Sequence alignment of $E$. coli OMPLA and 15 homologous enzymes from other Gram-negative bacteria highlighting the conserved residues in the active site, with the highly specific consensus sequence motif (YTQ- $\mathrm{X}_{n}-\mathrm{G}-\mathrm{X}_{2}-\mathrm{H}-\mathrm{X}-\mathrm{SNG}$ ) by Snijder et al. (2001) (reproduced with permission from the publisher: http://www.elsevier.com/). Residues involved in calcium binding are indicated above the alignment by open circles for theL3L4 site and black circles for the catalytic site. The activesite residues are indicated by the black arrowheads below the sequences. Strictly conserved residues are shown in black boxes, homologous residues in grey boxes, Residues involved in substrate binding and dimerization are indicated by the lower-case letters below the alignment: substrate binding (s), dimerization (d), and both substrate binding and dimerization (b). 
from the 'normal' L-variant, with less than $2 \%$ lysophospholipids, to the 'lyso' S-variant, with over $50 \%$ lysophospholipids in the membrane (Bukholm et al., 1997). The 'lyso' variant is more haemolytic, with enhanced affinity for epithelial cells and increased release of urease and VacA toxin. The deletion of the pldA gene in the S-variant restored the normal phenotype, suggesting that the L- to $S$ - transition is due to the activation of OMPLA (Bukholm et al., 1997). In an extension of this study Tannaes et al. (2001) indicated that the change in membrane lipid composition is actually due to phase variation in the pldA gene. They showed that a change in the C-tract length of this gene results in reversible frame-shifts, translation of a full-length or truncated pldA, and the production of active or inactive OMPLA. Furthermore, growth at low $\mathrm{pH}$ resulted in a selection of the bestadapted (S) phenotype, with an almost complete conversion of the normal L-variant (C7, OMPLA 'off) to the lyso S-variant (C8, OMPLA 'on'). The observed difference in colony morphology could not be explained by an LPS variation; therefore it was concluded that the pldA gene might contribute to survival of $H$. pylori at low $\mathrm{pH}$ (Tannaes et al., 2001). More recently, Tannaes et al. (2005) investigated the significance of phase variation in the pldA gene of H. pylori and its association with peptic ulcer disease. The study suggested that OMPLA activity is a virulence factor in H. pylori, as isolates with high OMPLA activity are significantly associated with patients suffering from peptic ulcer. The study also suggested a two-step regulation of OMPLA in H. pylori: (i) phase variation in the homopolymorphic tract of the pldA gene from 'off' to 'on' and (ii) protein level regulation among the pldA 'on' bacterial cells (Tannaes et al., 2005). Salaun et al. (2005) investigated 31 phase-variable genes in $H$. pylori following murine gastric colonization for up to one year in three unrelated $H$. pylori strains. They concluded that genes which are most likely to be associated with immediate niche fitting, including the pldA gene and LPS biosynthesis genes, changed more rapidly than other surface-protein genes which may be under control of the adaptive immune system. These findings suggest a role for these genes in determining initial fitness for colonization of $H$. pylori as well as in subsequent niche adaptation (Salaun et al., 2005). Moreover, Berstad et al. (2002) suggested that the $\mathrm{pH}$-activated phosoholipase $\mathrm{A}_{2}$ in $\mathrm{H}$. pylori acts as an initial mucosal barrier breaker and is considered to have a pivotal role in the pathogenesis of peptic ulcer. The research also indicated that the gastric juice of $H$. pyloriinfected individuals contains significantly higher $\mathrm{PLA}_{2}$ and lyso-PC concentrations than that of healthy individuals (Berstad et al., 2002).

Neisseria spp. The presence of outer-membraneassociated phospholipase activity in $N$. gonorrhoeae was first reported by Wolf-Watz et al. (1975). Later Senff et al. (1976) reported that this activity was calcium dependent, with a $\mathrm{pH}$ optimum of $8 \cdot 0$ to $8 \cdot 5$, and was sensitive to detergents, such as $0 \cdot 1 \%$ Triton X-100. The presence of a putative pldA gene was also demonstrated in the genome sequences of the human pathogens $N$. meningitidis and $N$. gonorrhoeae, indicating that the deduced meningococcal and gonococcal OMPLA proteins differ in only a few amino acid residues (Brok et al., 1998). Recently, Bos et al. (2005) studied the presence and conservation of OMPLA among meningococcal strains and tested its immunogenicity in an animal model. The vaccine potential of OMPLA in this human pathogen was also investigated, as a high level of conservation of OMPLA is maintained among meningococcal strains. However, the study showed that OMPLA is expressed in vitro by both pathogenic and non-pathogenic neisserial strains. The investigators demonstrated that OMPLA functions as an autolysin that acts under specific conditions, such as prolonged growth of the bacteria. The vaccine potential of the protein was investigated by immunizing mice with in vitro refolded, recombinant OMPLA. Although high levels of antibody to the OMPLA were obtained, the murine sera were neither bactericidal nor protective. Also, convalescent patients and vaccine sera did not contain detectable levels of antiOMPLA antibodies, indicating that OMPLA may not be sufficiently immunogenic to be included in a meningococcal vaccine (Bos et al., 2005).

Yersinia spp. $Y$. enterocolitica produces a virulenceassociated $\mathrm{PLA}_{2}$ (YplA) that is secreted via its flagellar type III secretion apparatus (Young et al., 1999). YplA appears to be closely related to PhlA of S. liquefaciens. However, initial reports suggested that the native form of YplA was 59 amino acids shorter than PhlA (Schmiel et al., 2000). The role of YplA in Y. enterocolitica pathogenesis has not been fully described, but this enzyme is proposed to contribute to increased inflammation in mesenteric lymph nodes during Yersinia infection (Schmiel et al., 1998). The pldA gene in Y. pseudotuberculosis encodes a phospholipase that is active towards both phosphatidylcholine and sphingomyelin, which are the major phospholipid components of the outer leaflet of eukaryotic cell membranes. The determined 1468 bp sequence, including the pldA gene with flanking regions, was found to be $100 \%$ identical to the corresponding sequence of $Y$. pestis, the causative agent of human plague (Karlyshev et al., 2001). Moreover, the researchers indicated that a PldA mutant strain of $Y$. pseudotuberculosis exhibited reduced phospholipase activity compared to the wild-type strain and confirmed in vivo attenuation of the mutant, therefore suggesting that PldA is an essential virulence factor in the murine yersiniosis model of infection (Karlyshev et al., 2001).

Legionella spp. Two PLA activities secreted by several Legionella spp. were considered as possible powerful agents of legionellae in causing lung disease due to destruction of lung surfactant and epithelial cells (Flieger et al., 2000). L. pneumophila secretes, via its type II secretion system, PLA activities that are distinguished by their specificity for certain phospholipids. However, the timing of PLA and lysophospholipase A secretion in L. pneumophila differed as compared to other bacterial species. PLA activity 
was secreted prior to lysophospholipase A activity in $L$. pneumophila, which may lead to an accumulation of the cytotoxic agent (Flieger et al., 2001). The L. pneumophila plaA gene, encoding a 309 amino acid protein (PlaA) that cleaves fatty acids from lysophospholipids, was identified and characterized by Flieger et al. (2002). Overexpression of plaA completely protected L. pneumophila from the toxic effects of lysophosphatidylcholine, suggesting a role for PlaA in bacterial detoxification of lysophospholipids. By screening a genomic library of L. pneumophila, Flieger et al. (2004) found an additional L. pneumophila gene, plaB, which encoded a cell-associated haemolytic activity and contained a lipase consensus motif in its deduced protein sequence. L. pneumophila $\mathrm{PlaB}$ shares several properties with the OMPLA of various enteropathogenic bacteria, including E. coli and S. typhimurium, and other Gram-negative bacteria, including $H$. pylori or N. meningitidis. Flieger et al. (2004) generated isogenic L. pneumophila plaB mutants and tested them for haemolysis, lipolytic activities and intracellular survival in amoebae and macrophages. Compared to the wild-type, the plaB mutant showed reduced haemolysis of human red blood cells and almost completely lost its cell-associated lipolytic activity. Therefore it was concluded that $L$. pneumophila plaB is the gene encoding the major cell-associated PLA, possibly contributing to bacterial cytotoxicity due to its haemolytic activity. On the other hand, in view of the fact that the plaB mutant multiplied like the wild-type both in U937 macrophages and in Acanthamoeba castellanii amoebae, plaB is not essential for intracellular survival of the pathogen (Flieger et al., 2004).

Campylobacter spp. OMPLA is considered to be a major haemolytic factor in C. coli. Grant et al. (1997) identified a pldA gene upstream of an operon encoding an enterochelin transport system in C. coli. The pldA gene product is a $35 \mathrm{kDa}$ protein with significant similarity to the $E$. coli OMPLA. The recombinant protein displayed calciumdependent lysophospholipase and phospholipase activities in vitro, indicating similar expression and recognition of this gene product by E. coli. Furthermore, a constructed pldA mutant in C. coli showed reduced haemolytic activity compared to the wild-type strain, indicating a role for PLA in the lysis of erythrocytes and suggesting a possible role for PLA in virulence of Campylobacter species. A pldA gene sequence was also reported for $C$. jejuni, encoding a putative PLA protein with significant similarity to PLA in C. coli (Brok et al., 1998). Istivan et al. (2004) reported a membrane-bound haemolytic PLA activity in Campylobacter concisus strains isolated from gastroenteritis cases in children and also in two C. concisus reference strains, ATCC 51561 and ATCC 51562. The cell-associated PLA was extracted and partially purified and was found to be a heat-stable, calcium-dependent PLA with both haemolytic and cytolytic effects on different types of erythrocytes and on tissue culture cell lines respectively (Istivan et al., 2004). The sequence of the pldA gene in C. concisus strain RCH3 (GenBank accession no. AJ786391) was analysed, and the translated 297 amino acid sequence for this gene product (Q6A175) has a predicted molecular mass of $35.076 \mathrm{kDa}$ (www.ebi.uniprot.org/uniprot-srv). A BLAST search performed on Q6A175 showed that it has 97\% identity to the amino acid sequence for PLA in C. coli (O32349) and $76 \%$ identity to PLA of C. jejuni (Q9PMU8), while it has only $34 \%$ identity to the OMPLA sequence of E. coli (Q8CVJ6) (Fig. 5). A three-dimensional structure for the monomeric form of OMPLA in C. concisus and C. coli showing the high similarity between the beta-barrel molecules in these two species is illustrated in Fig. 6. The $\beta$-barrel structure of OMPLA in Campylobacter spp. has a significant resemblance to the structure of OMPLA protein molecules of other enteric Gram-negative bacteria.

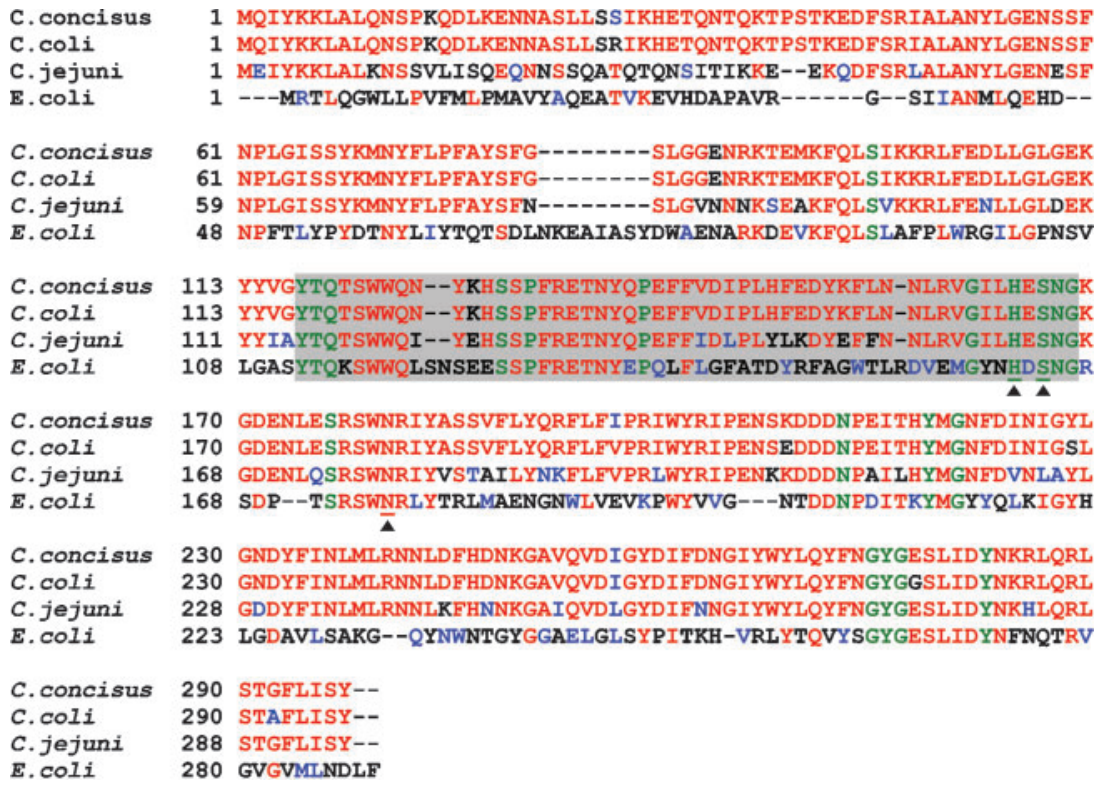

Fig. 5. Alignment and analysis of OMPLA sequences in C. concisus, C. coli, C. jejuni and E. coli. The green colour indicates the strictly conserved residues among $p / d A$ gene sequences of Gram-negative bacteria. The black arrowheads indicate the active sites in OMPLA sequences. The grey highlighted region indicates the consensus sequence motif for this family of phospholipases (YTQ- $\mathrm{X}_{\mathrm{n}}-\mathrm{G}-\mathrm{X}_{2}-\mathrm{H}-\mathrm{X}-\mathrm{SNG}$ ). 

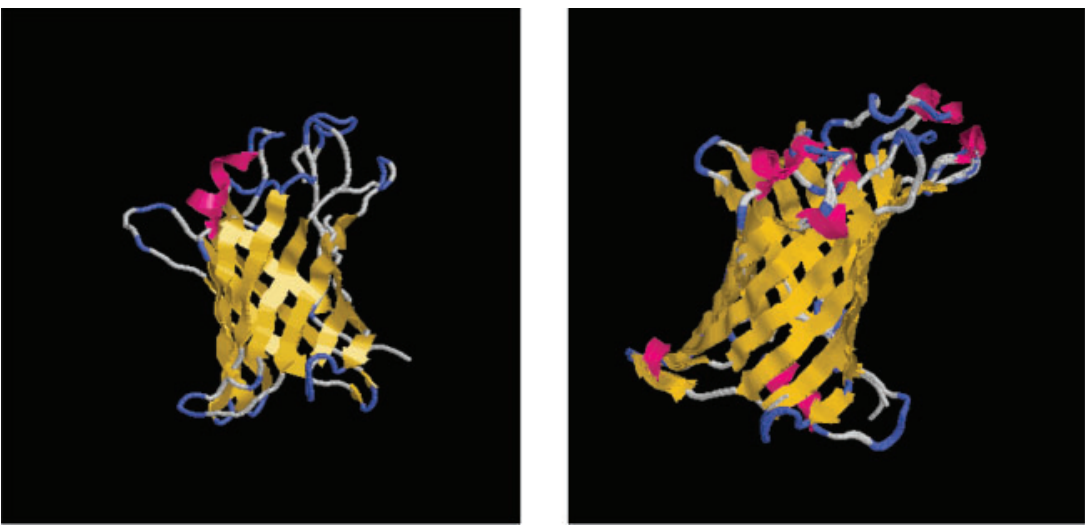

Fig. 6. The $\beta$-barrel protein molecule structure for a PLA monomer of C. concisus (left), and C. coli (right). The 3D protein structure for the PLA molecule was constructed using the 3-D JIGSAW Protein comparative modelling server. RasTop 1.3 by Philippe Valadon (2000) and RasMol molecular graphics programs were used for visualization and to create the $3 D$ representation of the PLA molecule.

The genome sequence of $C$. jejuni NCTC 11168 (Parkhill et al., 2000) revealed the presence of a pldA gene sequence (Cj1351) upstream of the ceuB and ceuC genes, similar to the position of the pldA gene in the C. coli genome (Grant et al., 1997) and in the C. concisus genome (T. S. Istivan, unpublished data). The ability of pldA mutant strains in $C$. jejuni to serve as viable bacterial vaccines against caecal colonization was investigated by Ziprin et al. (2001). They found that $C$. jejuni strains containing mutations in the $c i a B$ and pldA genes were extremely poor colonizers, and were avirulent as a result of their impaired ability to colonize the chicken caecum. However, although the mutants proved avirulent, inoculation with these strains did not induce biologically significant resistance against subsequent challenge with the parental strain; this was in contrast to the $H$. pylori pldA mutant, which was unable to colonize mice but did induce a significant immune response (Dorrell et al., 1999; Ziprin et al., 2001).

\section{Conclusion}

Bacterial PLA is a key factor in bacterial-induced haemolysis. It is likely that this activity provides a favourable source of nutrients for bacterial growth in addition to providing a suitable environment for replication and survival. The pldA gene encoding OMPLA is highly conserved in Gramnegative bacteria, suggesting that it has a significant role in bacterial survival. However, the role of PLA in bacterial virulence is yet to be fully elucidated. In some species, such as $C$. jejuni, pldA gene mutants with no haemolytic activity are poor colonizers but do not provide protection against colonization with wild-type bacteria, suggesting that OMPLA may play a role in colonization but not necessarily in virulence. Conversely for $\mathrm{H}$. pylori, a PldA mutant did not colonize mice but did offer some protection against challenge, suggesting that for this highly specialized pathogen, in a unique niche, OMPLA is important in inducing pathogenesis.

Many Gram-negative bacteria possess OMPLA activity, including most of the species that are effective colonizers of mammals and birds. The presence of OMPLA enables the bacteria to modify the environment, often the intestine or respiratory tract, to enhance their survival in vivo. However, further research is required to identify the role of PLA in the pathogenesis and survival of Gram-negative bacteria.

\section{References}

Baaden, M., Meier, C. \& Sansom, M. S. P. (2003). A molecular dynamics investigation of mono and dimeric states of the outer membrane enzyme OMPLA. J Mol Biol 331, 177-189.

Berstad, A. E., Berstad, K. \& Berstad, A. (2002). pH-activated phospholipase $\mathrm{A}_{2}$ : an important mucosal barrier breaker in peptic ulcer disease. Scand J Gastroenterol 37, 738-742.

Bos, M., Tefsen, B., Voet, P., Weynants, V., van Putten, J. P. \& Tommassen, J. (2005). Function of neisserial outer membrane phospholipase A in autolysis and assessment of its vaccine potential. Infect Immun 73, 2222-2231.

Brok, R. G., Brinkman, E., Boxtel, R., Bekkers, A. C., Verheij, H. M. \& Tommassen, J. (1994). Molecular characterization of enterobacterial pldA genes encoding outer membrane phospholipase A. J Bacteriol 176, 861-870.

Brok, R. G., Boots, A. P., Dekker, N., Verheij, H. M. \& Tommassen, J. (1998). Sequence comparison of outer membrane phospholipases A: implications for structure and for the catalytic mechanism. Res Microbiol 149, 703-710.

Bukholm, G., Tannaes, T., Nedenskov, P., Esbensen, Y., Grav, H. J. \& Hoving, T. (1997). Colony variation of Helicobacter pylori: pathogenic potential is correlated to cell wall lipid composition. Scand J Gastroenterol 32, 445-454.

de Geus, P., van Die, I., Bergmans, H., Tommassen, J. \& de Haas, G. (1983). Molecular cloning of pldA, the structural gene of outer membrane phospholipase of E. coli K12. Mol Gen Genet 190, 150-155. Dekker, N. (2000). Outer-membrane phospholipase A: known structure, unknown biological function. Mol Microbiol 35, 711-717.

Doi, O., Ohki, M. \& Nojima, S. (1972). Two kinds of phospholipase A and lysophospholipase in Escherichia coli. Biochem Biophys Acta 260, 244-258.

Dorrell, N., Martino, M. C., Stabler, R. A., Ward, S. J., Zhang, Z. W., McColm, A. A., Farthing, M. J. G. \& Wren, B. W. (1999). Characterization of Helicobacter pylori PldA, a phospholipase with a role in colonization of the gastric mucosa. Gastroenterology 117, 1098-1104.

Farber, S. A., Olson, E. S., Clark, J. D. \& Halpern, M. E. (1999). Characterization of $\mathrm{Ca}^{2+}$-dependent phospholipase $\mathrm{A}_{2}$ activity during zebrafish embryogenesis. J Biol Chem 274, 19338-19346. 
Flieger, A., Gong, S., Faigle, M., Deeg, M., Bartmann, P. \& Neumeister, B. (2000). Novel phospholipase activity secreted by Legionella species. J Bacteriol 182, 1321-1327.

Flieger, A., Gong, S., Faigle, M., Northoff, H. \& Neumeister, B. (2001). In vitro secretion kinetics of proteins from Legionella pneumophila in comparison to proteins from non-pneumophila species. Microbiology 147, 3127-3134.

Flieger, A., Neumeister, B. \& Cianciotto, N. (2002). Characterization of the gene encoding the major secreted lysophospholipase A of Legionella pneumophila and its role in detoxification of lysophosphatidylcholine. Infect Immun 70, 6094-6106.

Flieger, A., Rydzewski, K., Banerji, S., Broich, M. \& Heuner, K. (2004). Cloning and characterization of the gene encoding the major cell-associated phospholipase A of Legionella pneumophila, plaB, exhibiting hemolytic activity. Infect Immun 72, 2648-2658.

Ghannoum, M. A. (2000). Potential role of phospholipases in virulence and fungal pathogenesis. Clin Microbiol Rev 13, 122-143.

Givskov, M., Olsen, L. \& Molin, S. (1988). Cloning and expression in Escherichia coli of the gene for extracellular phospholipase $A_{1}$ from Serratia liquefaciens. J Bacteriol 170, 5855-5862.

Grant, K. A., Belandia, I. U., Dekker, N., Richrdson, P. T. \& Park, S. F. (1997). Molecular characterization of pldA, the structural gene for a phospholipase A from Campylobacter coli, and its contribution to cell-associated hemolysis. Infect Immun 65, 1172-1180.

Homma, H., Kobayashi, T., Chiba, N. \& 7 other authors (1984). The DNA sequence encoding pldA gene, the structural gene for detergentresistant phospholipase A of E. coli. J Biochem 96, 1655-1664.

Horrevoets, A. J., Hackeng, T. M., Verheij, H. M., Dijkman, R. \& de Haas, G. H. (1989). Kinetic characterization of Escherichia coli outer membrane phospholipase A using mixed detergent-lipid micelles. Biochemistry 28, 1139-1147.

Istivan, T. S., Coloe, P. J., Fry, B. N., Ward, P. \& Smith, S. C. (2004). Characterization of a haemolytic phospholipase A2 activity in clinical isolates of Campylobacter concisus. J Med Microbiol 53, 483-493.

Karlyshev, A. V., Oyston, P. C., Williams, K., Clark, G. C., Titball, R. W., Winzeler, E. A. \& Wren, B. W. (2001). Application of highdensity array-based signature-tagged mutagenesis to discover novel Yersinia virulence-associated genes. Infect Immun 69, 7810-7819.

Kim, M. K. \& Rhee, J. S. (1996). Purification and biochemical properties of extracellular phospholipase $A_{1}$ from Serratia sp. MK1. J Microbiol Biotechnol 6, 407-413.

Kingma, R. L. \& Egmond, M. R. (2002a). Substrate interferes with dimerisation of outer membrane phospholipase A. FEBS Lett 516, 31-34.

Kingma, R. L. \& Egmond, M. R. (2002b). Activation of a covalent outer membrane phospholipase A dimer. Eur J Biochem 269, 2178-2185.

Kingma, R., Fragiathaki, M., Snijder, H., Dijkstra, B., Verheij, H., Dekker, N. \& Egmond, M. (2000). Unusual catalytic triad of Escherichia coli outer membrane phospholipase A. Biochemistry 39, 10017-10022.

Kingma, R. L., Snijder, H. J., Bauke, W., Dijkstra, B. W., Dekker, N. \& Egmond, M. R. (2002). Functional importance of calcium binding sites in outer membrane phospholipase A. Biochem Biophys Acta 1561, 230-237.

Matoba, Y. \& Sugiyama, M. (2003). Atomic resolution structure of prokaryotic phospholipase $\mathrm{A}_{2}$ : analysis of internal motion and implication for a catalytic mechanism. Proteins 51, 453-469.

Matoba, Y., Katsube, Y. \& Sugiyama, M. (2002). The crystal structure of prokaryotic phospholipase A . J Biol Chem 277, 20059-20069.

Merck, K., de Cock, H., Verheij, H. \& Tommassen, J. (1997). Topology of the outer membrane phospholipase A of Salmonella typhimurium. J Bacteriol 179, 3443-3450.
Nilius, M. \& Malfertheiner, P. (1996). Helicobacter pylori enzymes. Aliment Pharmacol Ther 10, 65-71.

Parkhill, J., Wren, B. W., Mungall, K. \& 18 other authors (2000). The genome sequence of the food-borne pathogen Campylobacter jejuni reveals hypervariable sequences. Nature 403, 665-668.

Rock, C. O., Jackowski, S. \& Cronan, J. E. (1996). Lipid metabolism in prokaryotes. In Biochemistry of Lipids, Lipoproteins and Membranes, pp. 35-74. Edited by D. E. Vance \& J. Vance. Amsterdam: Elsevier.

Salaun, L., Ayraud, S. \& Saunders, N. J. (2005). Phase variation mediated niche adaptation during prolonged experimental murine infection with Helicobacter pylori. Microbiology 151, 917-923.

Scandella, C. J. \& Kornberg, A. (1971). A membrane-bound phospholipase $\mathrm{A}_{1}$ purified from Escherichia coli. Biochemistry 10, 4447-4456.

Schmiel, D. H. \& Miller, V. L. (1999). Bacterial phospholipases and pathogenesis. Microbes Infect 1, 1103-1112.

Schmiel, D. H., Wagar, E., Karamanou, L., Weeks, D. \& Miller, V. L. (1998). Phospholipase A of Yersinia enterocolitica contributes to pathogenesis in a mouse model. Infect Immun 66, 3941-3951.

Schmiel, D. H., Young, G. M. \& Miller, V. L. (2000). The Yersinia enterocolitica phospholipase gene $y p l A$ is part of the flagellar regulon. J Bacteriol 182, 2314-2320.

Senff, L. M., Wegener, W. S., Brooks, G. F., Finnerty, W. R. \& Makula, R. A. (1976). Phospholipid composition and phospholipase A activity of Neisseria gonorrhoeae. J Bacteriol 127, 874-880.

Snijder, H. J. \& Dijkstra, B. W. (2000). Bacterial phospholipase A: structure and function of an integral membrane phospholipase. Biochim Biophys Acta 1488, 91-101.

Snijder, H. J., Ubarretxena-Belandia, I., Blaauw, M., Kalk, K. H., Verheij, H. M., Egmond, M. R., Dekker, N. \& Dijkstra, B. W. (1999). Structural evidence for dimerization-regulated activation of an integral membrane phospholipase. Nature 401, 717-721.

Snijder, H. J., Kingma, R. L., Kalk, K. H., Dekker, N., Egmond, M. R. \& Dijkstra, B. W. (2001). Structural investigations of calcium binding and its role in activity and activation of outer membrane phospholipase A from Escherichia coli. J Mol Biol 309, 477-489.

Snijder, H. J., Timmins, P. A., Kalk, K. H. \& Dijkstra, B. W. (2003). Detergent organisation in crystals of monomeric outer membrane phospholipase A. J Struct Biol 141, 122-131.

Song, J. K. \& Rhee, J. S. (2000). Simultaneous enhancement of thermostability and catalytic activity of phospholipase $A_{1}$ by evolutionary molecular engineering. Appl Environ Microbiol 66, 890-894.

Song, J. K., Kim, M. K. \& Rhee, J. S. (1999). Cloning and expression of the gene encoding phospholipase A1 from Serratia sp. MK1 in Escherichia coli. J Biotechnol 72, 103-114.

Songer, J. G. (1997). Bacterial phospholipases and their role in virulence. Trends Microbiol 156, 156-161.

Tannaes, T., Dekker, N., Bukholm, G., Bijlsma, J. \& Appelmelk, B. J. (2001). Phase variation in the Helicobacter pylori phospholipase A gene and its role in acid adaptation. Infect Immun 69, 7334-7340.

Tannaes, T., Bukholm, I. K. \& Bukholm, G. (2005). High relative content of lysophospholipids of Helicobacter pylori mediates increased risk for ulcer disease. FEMS Immunol Med Microbiol 44, 17-23.

Ubarretxena-Belandia, I., Boots, J. W., Verheij, H. M. \& Dekker, N. (1998). Role of the cofactor calcium in the activation of outer membrane phospholipase A. Biochemistry 37, 16011-16018.

Waite, M. (1996). Phospholipases. In Biochemistry of Lipids, Lipoproteins and Membranes, pp. 211-236. Edited by D. E. Vance \& J. Vance. Amsterdam: Elsevier.

Wolf-Watz, H., Elmros, T., Normark, S. \& Bloom, G. D. (1975). Cell envelope of Neisseria gonorrhoeae: outer membrane and peptidoglycan 
composition of penicillin-sensitive and -resistant strains. Infect Immun 11, 1332-1341.

Xerry, J. \& Owen, R. J. (2001). Conservation and microdiversity of phospholipase A (pldA) gene of Helicobacter pylori infecting dyspeptics from different countries. FEMS Immunol Med Microbiol 32, 17-25.

Young, G. M., Schmiel, D. H. \& Miller, V. L. (1999). A new pathway for the secretion of virulence factors by bacteria: the flagellar export apparatus functions as a protein-secretion system. Proc Natl Acad Sci U S A 96, 6456-6461.

Zhang, Z. W., Dorrell, N., Wren, B. W. \& Farthing, M. J. G. (2002). Helicobacter pylori adherence to gastric epithelial cells: a role for nonadhesin virulence genes. J Med Microbiol 51, 495-502.

Ziprin, R. L., Young, C. R., Byrd, J. A., Stanker, L. H., Hume, M. E., Gray, S. A., Kim, B. J. \& Konkel, M. E. (2001). Role of Campylobacter jejuni potential virulence genes in cecal colonization. Avian Dis 45, 549-557. 